Session 2520

\title{
Neural Networks and Fuzzy Systems: A Relationship Between Them
}

\author{
E. J. Mastascusa \\ Electrical Engineering Department \\ Bucknell University \\ Lewisburg, PA 17837
}

\section{Introduction}

Neural networks and fuzzy systems have much in common, but can be approached and taught as though they were completely disparate subjects. Explicit links between the two subjects can aid students as they grapple with both subjects'. Shared structures and concepts explicitly presented can be an aid to learning.

In this paper we will present a bridge between the two subject areas. We propose that this bridge is a useful paradigm that helps students learning fuzzy systems and neural networks.

\section{The Neural Network}

We begin by considering a three layer neural network. The general structure of such a network is given in Figure 1, which emphasizes the inputs and the input layer of neurons.

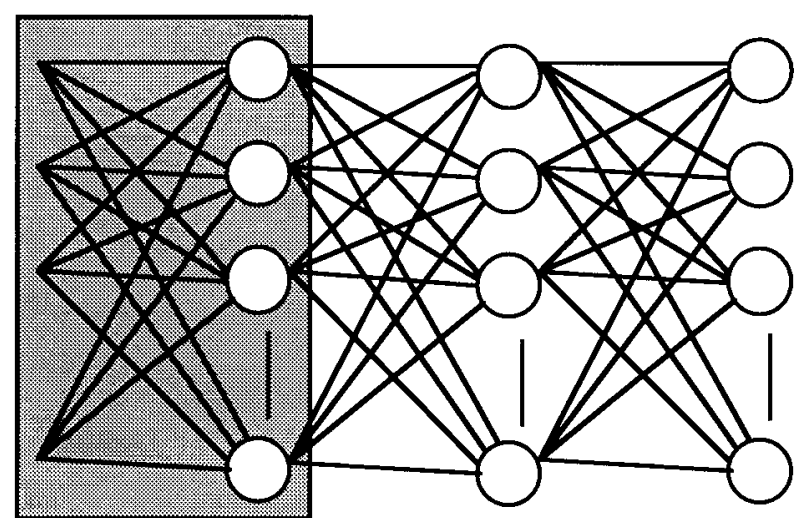

Inputs and Input Layer

Figure 1

A Three Layer Neural Network Structure

This particular structure, a three layer feedforward neural network is a starting point for other, more complex, neural network structures. However, this particular network is related to a fuzzy system because this network implements a rule base for a fuzzy system and the rule base can be determined from an examination of the network and the weights associated with the network connecting links. This link between the neural network and an associated fuzzy system rule base is not presented in either neural network texts or fuzzy systems texts. 
Several observations lead to this conclusion about this link,

A. The inputs to the network can take on any real value, positive or negative.

B. The weights of the connecting links can take on any real value, positive or negative.

C. The internal signals, and the outputs can only take on values between zero and one.

In this list, $\mathrm{A}$ and $\mathrm{B}$ imply that the input layer determines the truth level, in a fuzzy system sense, of statements about the input variables, and $\mathrm{C}$ implies a set of logical statements that use the truth levels at the outputs of the first layer. We will consider these two stages separately.

\section{The Input Layer}

Each neuron in the input layer determines, as its output, the truth of a statement:

Quality $Q_{n}$ is true.

The quality, Qn, is a "composite" quality determined by the weights in the first (input) layer. What is meant by a "composite" quality can best be understood with an example. The specific example we choose is a "large" building, an example that can be visualized easily by students.

Let's look at how we could measure the truth of the statement "Building A is large." Here, "large" is the composite quality that could be measured by a neuron with inputs of building height, area and/or volume. We could agree that buildings over 200 feet tall are large and buildings that cover more than an acre are large but there are large buildings that have a combination of height and area that makes them large without satisfying either the height or area condition singly. We can account for that, for example, by considering any building with a combination of height and area above the line in Figure 2 to be a large building. If we consider the statement "This building is large" to be true when the building parameters fall above the line, and false when the building parameters place it below the line, then we have a "crisp" logic representation for what a "large" building is.

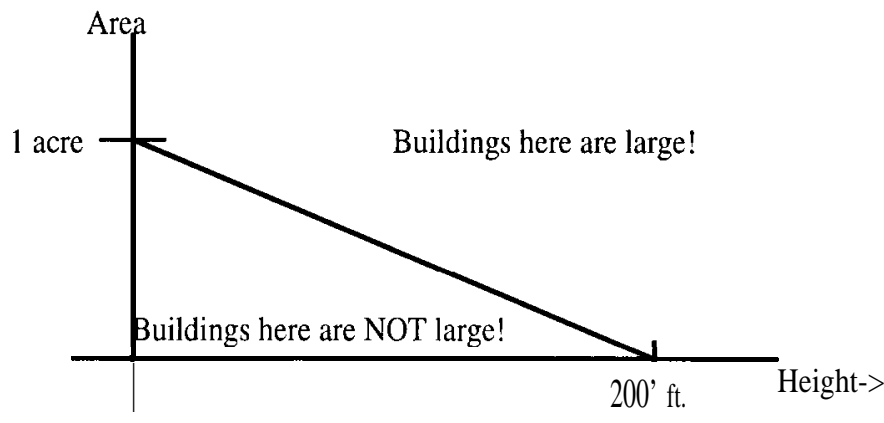

Figure 2

Graphical Representation of "Large"

A statement about whether a building is large or not can also be put into a fuzzy form. For example, buildings close to the line in Figure 2 are marginal in some sense, and we may not wish to consider them as having a high membership level in the set of large buildings. Alternatively, we would not say that the truth value of the statement "This building is large. " is high if the building's parameters fall close to the line. We would consider the statement to be false when the building's parameters fell well below the line.

A neuron that computes membership levels in the set of tall buildings is shown in Figure 3 . If a building's parameters fall exactly on the line in Figure 2, then the net input to the neuron is zero, and for most membership functions for $\mathrm{f}(\mathrm{net}$ ) (a sigmoid function for example), the output will be one-half at that point. 


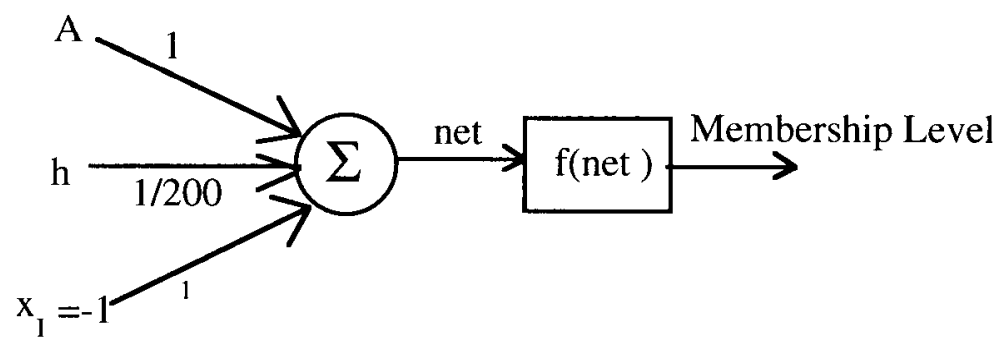

Figure 3

A Neuron Circuit for Computing Membership Level

in the Set of Large Buildings

Now, the entire first layer of neurons in the three layer structure can be thought of as measuring numerous composite qualities like "large" in our building example. Although there may not be a predefine name for those qualities that are actually computed by the network, we can still think in terms of those qualities and we can consider the output of each neuron as computing the truth of the statement "Quality $Q_{n}$ is true." The computed truth value lies somewhere between zero and one when a sigmoidal function is used for $\mathrm{f}(\mathrm{net})$. Indeed, past the input layer, all signals would then lie between zero and one and the rest of the three layer neural network structure can be thought of as a fuzzy logic circuit.

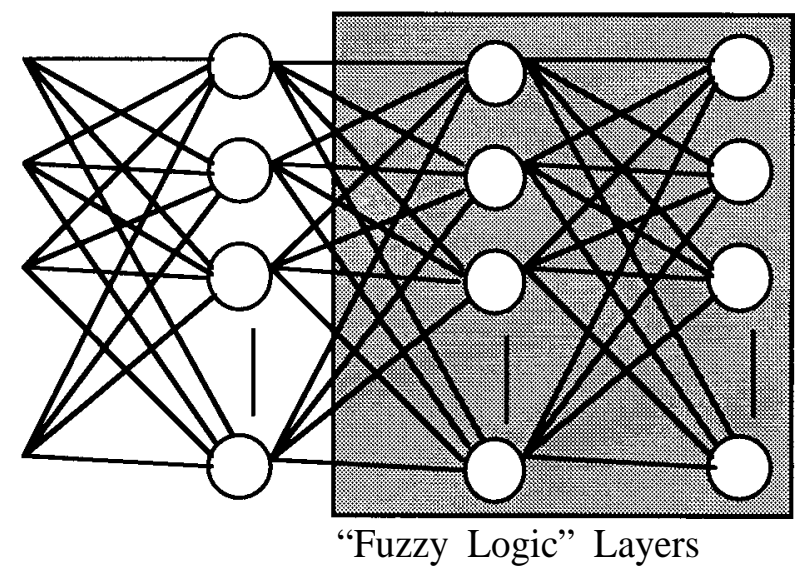

Figure 4.

Emphasizing the Logic Circuitry

Let us examine the "logic" portion of the neural network. That is the shaded area in Figure 4. Let us imagine replacing all of the sigmoidal functions with step functions. In the step functions we have:

$$
\begin{aligned}
& \mathrm{f}(\text { net })=1 \text { for net }>0 \\
& \mathrm{f}(\text { net })=0 \text { otherwise. }
\end{aligned}
$$

Note that we imagine replacing all sigmoidal functions with steps including the functions in the input layer. After that replacement, the inputs to the last two layers are all classical, crisp logic signals, that is either zero (0) or one (1). The last two layers of neurons then, in fact, forma classical, crisp logic circuit. The inputs to this logic circuit are the truth values $\left(\mathrm{O}\right.$ or 1 ) associated with each input quality statement, that is "Quality $Q_{n}$ is true." and the outputs of the complete neural network are Boolean functions of those quality statements. Given that, the output can be phrased as:

$$
\text { Outputi }=F i\left(Q_{1}, Q_{2}, \ldots Q_{N}\right)
$$


Since each Boolean output function can be expressed in terms of minterms, this becomes:

Outputi is TRUE IF $\left(\mathrm{Q}_{1}\right.$, is TRUE and $\mathrm{Q}_{2}$ is FALSE...) OR ( etc. ....)

The complete set of output statements then constitutes a rule base that can be expressed in the standard form for a rule base, that is a set of conditional statements that tell when the output is "True" (as in "IF the statement "The Temperature is Cold" is TRUE, AND the statement "The temperature is Falling. " is TRUE, then the High Heat Input is TRUE. ").

\section{Conclusions}

In this paper we have explored a simple way to move from a particular type of neural network to an "equivalent" fuzzy system which performs in the same manner as the neural network. The equivalent fuzzy system has a rule base that is extracted directly from the weights associated with the various layers of the neural network and the structure of the neural network. The rule base for the fuzzy system depends upon composite qualities derived from weight relationships within the neural network.

This connection between an equivalent fuzzy system and the original neural network is an aid to student comprehension of both systems. The resultant fuzzy system rule base lends itself to straight-forward implementation in a structure similar to that of the original neural network.

\section{References}

1. Kosko, B., "Fuzzy Thinking, The New Science of Fuzzy Logic", Hyperion, Inc., New York, NY, 1993, pp. 205-214.

2. McNeill, D. and Freiberger, P., "Fuzzy Logic”, Simon \& Schuster, New York, NY, 1993, pp. 229-232.

3. Zurada, J. M., "Introduction to Artifical Neural Systems”, West Publishing Co., St. Paul, MN, 1992, pp. 31-34.

4. Kosko, B., “Neural Networks and Fuzzy Systems”, Prentice Hall, Englewood Cliffs, NJ, 1992, pp. 48-50.

\section{Biography}

E. J. MASTASCUSA, Professor of Electrical Engineering at Bucknell University, also taught at the University of Wyoming. His interests include development of an interdisciplinary computer-assisted controls laboratory, modelling and simulation in control systems, and development of electronic modules for introductory EE material. 